\title{
Time Matters: Effectiveness of Steaming Times on Forage for Animal Consumption
}

\author{
Leslie Serrano \\ Agriculture, Geosciences, and Natural Resources, University of Tennessee at Martin \\ Brehm Hall 253, Martin, TN 38238 \\ Tel: 731-881-7222Ｅmail: lesnserr@ut.utm.edu \\ Leslie Dodd \\ Agriculture, Geosciences, and Natural Resources, University of Tennessee at Martin \\ Brehm Hall 253, Martin, TN 38238 \\ Tel: 731-881-7222_Email: lesmdodd@ut.utm.edu \\ Bethanie Wood \\ Agriculture, Geosciences, and Natural Resources, University of Tennessee at Martin \\ Brehm Hall 253, Martin, TN 38238 \\ Tel: 731-881-7222_Email: betswood@ut.utm.edu \\ Jeremy D'Antoni
}

Agriculture, Geosciences, and Natural Resources, University of Tennessee at Martin Brehm Hall 253, Martin, TN 38238

Tel: 731-881-7222_Email: jdanto1@utm.edu

Joey Mehlhorn (Corresponding Author)

Agriculture, Geosciences, and Natural Resources, University of Tennessee at Martin

Brehm Hall 254, Martin, TN 38238

Tel: 731-881-7275_Ｅmail: mehlhorn@utm.edu

Received: July 8, 2015

doi:10.5296/jas.v3i2.7946
Accepted: July 24, 2015

URL: http://dx.doi.org/10.5296/jas.v3i2.7946 


\begin{abstract}
The goal of this research is to determine the effectiveness of portable hay steamer equipment for horse owners averaging 1-15 head. Hay is a large portion of horses' diets, but it has a short shelf life. Dust from hay also becomes a problem due to respiratory diseases. There is a need for a healthier alternative for storing and feeding hay in order to promote optimum animal health. This study tests the effectiveness of four steaming times on the cleanliness of high-quality, Bermuda hay in the portable steamer. Swabs were taken from pre and post-steaming hay samples and incubated on nutrient agar plates at 37 degrees Celsius for 24 hours. After incubation, bacterial colonies were counted to compare the change in bacteria present from before and after the steaming process. Using a two-sample mean difference tests, we found steaming time had a significant impact on the cleanliness of hay.
\end{abstract}

Keywords: animal health, hay-steaming, Chronic Obstructive Pulmonary Disease (COPD)

\title{
1. Introduction
}

Hay is considered to be a large portion in the diets of horses due to the nutritional value of the forage (American Association of Equine Practitioners, 2002). Although hay is an important component, it has a short shelf life before quality begins to deteriorate or become susceptible to contaminants. Once a bale of hay sits for a period of time, bacteria will grow and mold will be apparent. Dust from hay also becomes a problem contributing to respiratory diseases (James, 2009). There is a need for a healthier alternative for storing and feeding hay in order to promote optimum animal health and well-being.

There are common respiratory diseases among species of animals similar to those in humans. A major factor contributing to the onset of illness or disease is the environment in which the animal lives. For most horses, the environment consists of a stable and pasture. Allergies developed in horses are often due to mold, dust, or airborne particles (Jean Hofve, 2007). Common sources of these include hay fed to the horse and straw used for bedding (Clarke, 1992). Mucus secretions induced by these particles will cause coughing and snorting, which will lead to inflammation and swelling. Airways may become partially blocked and smooth muscles constrict along the passage into the lungs resulting in excessive wheezing and coughing (Jean Hofve, 2007). Over time, this hypersensitive reaction reoccurs as a condition called Chronic Obstructive Pulmonary Disease, COPD (Blackman \& Moore-Colyer, 1998).

COPD accounts for half of the lung diseases present in performance horses. COPD is also referred to as "heaves", "broken wind", and "equine asthma" due to the labored breathing seen in animals affected (PM, DI, \& BC, 1995). "Farmer's lung" is a human disease often compared to COPD in horses due to their similarities, which also is associated with inhaling dust from moldy hay (Gregory \& Lacey, 1963). In matters of quality, allergen-containing particles do not discriminate. All types of hay (even that of excellent quality) contain bacteria, mold, and fungal spores, which cannot be seen by the naked eye (Eduard, Lacey, Karlsson, Palmgren, \& Blomquist, 1990). A damp and humid environment provides perfect conditions for bacteria to thrive, further contaminating the air. Figure 1 shows the distribution of mold spores in hay fed to animals in the United States. While in the stable, horses continually inhale particles, which can develop or worsen existing COPD (Jean Hofve, 2007). 
Although hay is a vital feedstuff in animal nutrition, the pathogens it contains are hazardous to the health of animals (as well as owners) and must be managed. Improving ventilation of the stable or barn is limited in its effects on actually producing "cleaner air". Blackman and Moore-Colyer explain that improved ventilation does not eliminate the dust particles from the animal's breathing zone. In order to improve the quality of air, the source of the particles must be changed (Blackman \& Moore-Colyer, 1998). Soaking hay for a short period of time in order to eliminate dust is a practice adopted by some horse owners. However, soaking hay nets have been observed to leach nutrients (Blackman \& Moore-Colyer, 1998) and produce large amounts of wastewater (Warr \& Petch, 1992). In a study comparing soaked hay to steamed hay, Blackman and Moore-Colyer described the use of steaming hay as an alternative to soaking due to its ability to reduce the amount of hazardous particles (Blackman \& Moore-Colyer, 1998). A more recent study suggests that the HayGain Hay Steamer is effective at significantly reducing dust particles as well as producing a palatable product that does not alter an animal's feed intake (Moore-Colyer \& James, 2009).

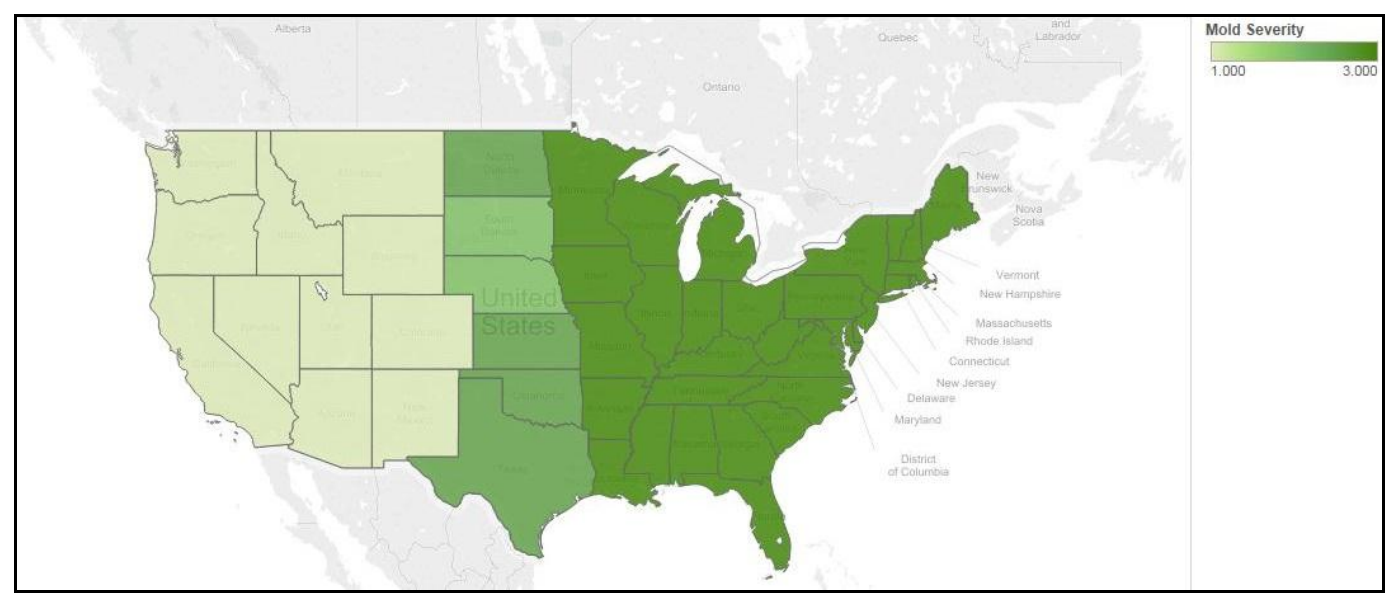

Figure 1. Mold distribution in the United States during summer months (Jean Hofve, 2007).

The goal of this research is to determine the overall effectiveness of portable hay steamer equipment for equine owners and the appropriate duration of steaming. The effectiveness of this product will impact the health of animals consuming sterilized hay. During this research, hay from two different bales was steamed using the HayGain portable hay steamer. Four trials were conducted with steaming times of: 45, 60, 75, and 90 minutes. Pre-steam and post-steam samples were collected. Bacteria colonies were counted and pre samples were compared to post samples to determine the effectiveness of the steamer at different time intervals. Elimination of the majority of bacteria colonies was expected after steaming.

\section{Materials and Methods}

Two bales of Bermuda hay were obtained from the same source to limit variability of different grades of hay. In order to compare water gain to bacterial growth in the bales of hay, the half-bales were weighed both before and after steaming. For the first trial, half of the first bale was weighed and the measure was recorded. 
Maintaining a systematic, clean collection and plating procedure was of the utmost importance. This was achieved through various applications of aseptic technique to minimize contamination. Samples from the bale needed to be from different locations within the bale in order to observe effectiveness of steam throughout the whole bale. Three samples were taken from the half-bale by placing a sterile bag over the hand and reaching into the bale. Once the hay was grasped, the sterile bag was folded from the hand and sealed to reduce contamination from the steamer to the laboratory. The steamer was sanitized with alcohol solution applied by a clean washcloth. This ensures that bacterial growth, mold, or contamination was present in the hay and not the steamer itself.

The half-bale of hay was placed into steamer bags and secured. After the HayGain steamer signals to start, the bale was steamed for desired durations of 45, 60, 75 and 90 minutes, respectively. Following steaming, the bale was weighed and the weight was recorded. Three more samples were taken using the same procedure outlined previously. Samples were delivered to the lab within ten minutes of taking the sample because bacterial growth can be achieved in approximately 20-30 minutes given optimal growth conditions. Following each trial, the steamer was rinsed with water and sanitized with isopropyl alcohol prior to the next use to maintain the same conditions for each trial.

For both pre and post samples, nutrient agar plates were inoculated using aseptic technique. The nutrient agar provided favorable ingredients needed for a number of different bacteria to grow. Students working in the lab twisted the cap off of a test tube filled with sterile water, and passed the opening of the test tube over hottest part of Bunsen burner flame (approximately 2-3 seconds) to achieve sterilization. A sterile swab was dipped into the sterile water and the tube is passed back through the flame and capped. Wet swab was used to swab the sample in desired sample bag and streak $1 / 4$ of the nutrient agar plate. Swab was discarded in a disinfectant solution to control a safe working environment. An inoculating loop was flame sterilized to avoid contamination from the environment and used to pull a new streak from the original $1 / 4$ of the plate. This was repeated twice until the full surface area of the plate was streaked.

Dilution of the original sample by this method provided a clearer view of bacterial colonies in order to count them. The final $1 / 4$ of the streaked plate was marked and was used to determine the bacterial colony count. This process is repeated for 15 pre and post samples taken for four trials providing a total of 30 observations for each time trial. A temperature of $37^{\circ} \mathrm{C}$ was chosen because this is approximately the internal temperature where growth would be likely to occur in horses' nostrils and lungs. Inoculated plates were incubated for 24 hours and colony counts were recorded. Pictures were taken to document results and data was entered into an Excel spreadsheet. Afterwards, all samples and plates were autoclaved to sterilize the materials, and were discarded safely.

\subsection{Data}

The four trials $(45,60,75$, and 90 minutes) produced 15 data points for pre-samples and 15 
data points for post-samples. This yielded a total sample size of 120 observations. These observations are listed below in Table 1 . This table provides the bacterial colony count for each trial, the mean colony count, and variance.

Table 1. Summary Colony Count Data for All Four Trials

\begin{tabular}{cccccccc}
\hline \multicolumn{2}{c}{$45-$-Minute Trial } & \multicolumn{2}{c}{ 60-Minute Trial } & \multicolumn{2}{c}{ 75-Minute Trial } & \multicolumn{2}{c}{90 -Minute Trial } \\
\hline Pre & Post & Pre & Post & Pre & Post & Pre & Post \\
Sample & Sample & Sample & Sample & Sample & Sample & Sample & Sample \\
Mean & Mean & Mean & Mean & Mean & Mean & Mean & Mean \\
17.27 & 1.53 & 0.47 & 0.67 & 0.73 & 0.0 & 15.27 & 4.27 \\
Variance & Variance & Variance & Variance & Variance & Variance & Variance & Variance \\
249.78 & 4.84 & 0.70 & 3.24 & 0.78 & 0.0 & 80.78 & 11.64 \\
\hline
\end{tabular}

Two bales of Bermuda hay were used. The 45 and 90-minute trials used bale 1, while the 60 and 75-minute trials used bale 2 . One aspect of the data that becomes immediately clear is the pre-sample data differs considerably across bales. To the naked eye, these bales appeared identical and were obtained through the same source. Pre-sample data indicated that the second bale had a considerably lower initial bacteria count.

From bale 1, the pre-sample mean bacteria count of the 45-minute trial was 17.27 and the 90-minute trial was 15.27. The mean bacteria count from bale 2 was considerably lower with a mean bacteria count for the 60 -minute trial of 0.47 and the 75 -minute trial was 0.73 . Based on these figures, it is expected that in Tables 1 also showed a higher variance for those trials from bale 1 compared to bale 2. Notably, the 90-minute trial did include one significant outlier. While the mean bacteria count for this trial's pre-sample was 15.27 , there was one observation that totaled 120 .

Post-sample data showed a reduction in the mean post-sample bacteria count for all but the 60-minute trial. From bale 1, the mean post-sample bacteria count for the 45-minute trial was 1.53 and the 90 -minute trial was 4.27. From bale 2, the mean post-sample bacteria count for the 60-minute trial was 0.67 and the 75 -minute trial was 0.00 . Again, we can see that the mean bacteria count associated with bale 2 was lower than that of bale 1 . Within the trials, Table 1 showed the 60-minute had one unusual observation of 7.

\subsubsection{Statistical Methods}

Based on the sample means and variances from Table 1, there is a significant difference in the means between the pre and post sample. Specifically, we want to determine whether steaming results in a reduction in bacteria count. Our testing hypothesis is

$$
\begin{aligned}
& H_{0}: \mu_{1} \leq \mu_{2} \\
& H_{1}: \mu_{1}>\mu_{2}
\end{aligned}
$$

where $\mu_{1}$ is the pre-sample population mean and $\mu_{2}$ is the post-sample population mean. The method by which we test this hypothesis is a two-sample difference of means test. 


\section{Macrothink}

Equation 2 shows the exact equation used in this computation:

$$
t=\frac{\bar{x}_{1}-\bar{x}_{2}}{\sqrt{\frac{s_{1}^{2}}{n_{1}}+\frac{s_{2}^{2}}{n_{2}}}}
$$

This method is a t-test that compares the pre-sample mean $\bar{x}_{1}$ to the post-sample mean $\bar{x}_{2}$. In the denominator, we find the sample variance $\left(\mathrm{s}^{2}\right)$ and sample size $\left(\mathrm{n}^{2}\right)$ for the pre and post samples. The degrees of freedom used determine the critical values for this test statistic are

$$
u /=\operatorname{Min}\left(n_{1}-1, \pi_{2}-1\right)
$$

Compared to other similar methods of testing mean differences, this method does not assume equal variances. This method was selected because it is the more conservative test statistic formula because it involves minimal assumptions and is the preferred method when working with small sample sizes. While we minimize the assumptions made when calculating the test statistic, we do make simplifying assumptions for the degrees of freedom. According to Doane and Seward (2007), equation 3 is a suitable approximation to the Welch-Satterthwaite adjusted degrees of freedom. We perform this test for each of our four trials containing 15 pre and post observations.

\subsubsection{Results}

Longer durations of steaming do not appear to significantly reduce the population of bacteria in our experiment. Trial four was the longest duration of steaming in our experiment (90 minutes) and we can conclude at $90 \%$ confidence that the bacteria colony count was reduced. The results from the remaining trials largely indicate improved performance of the steamer. Trials one (45 minutes) and three (75 minutes) indicate significant reductions in bacteria colony counts. In both trials, we can conclude at $99 \%$ confidence that steaming reduced the bacteria count in the hay. Relatively, trial one had a greater reduction in bacteria than trial three. This can be explained by the fact that bale one had a much larger population of bacteria prior to steaming, while bale two was a much cleaner bale of hay with pre samples showing very little to no bacteria present prior to steaming.

While trial one displayed the greatest mean difference in colony count, the hay was sampled from the bale that contained a larger population of bacteria. Therefore, there is greater opportunity for improvement with regards to cleanliness in trials one and four. Conversely, trial two (60 minutes) was the only trial that did demonstrate a statistically significant reduction in bacterial growth. Bale two was used for this trial and therefore allows for more limited improvements in cleanliness. The post-steaming sample for trial two indicated that the bacteria count remained near zero following one hour of steaming. With regards to time, it appears from our limited number of trials that there is a diminishing return to the duration of steaming following 75 minutes.

In addition to changes in bacteria count within time trials, we compared the impact of different steaming times on the same bale of hay. With this test, we compared the reduction in 
bacteria found in trials two and three and found evidence at $95 \%$ that steaming produced a greater reduction in bacteria at 75 minutes than 60 minutes in bale 2 . We also tested the change in bacteria count for the 60 and 90 minutes trials performed on bale 1. Surprisingly, we found evidence at $99 \%$ significance that the 60 -minute trial produced a larger reduction in bacteria count than the 90-minute trial.

Table 2. Results of Two-Sample Mean Difference Test and Critical Values

\begin{tabular}{lccc}
\hline & Mean & Variance & T-Statistic \\
\hline 45-Minute Trial & 17.27 & 1.53 & \\
$\quad$ Before & 249.78 & 4.84 & 3.82 \\
$\quad$ After & 0.47 & 0.70 & \\
60-Minute Trial & 0.67 & 3.24 & -0.39 \\
$\quad$ Before & & & \\
$\quad$ After & 0.73 & 0.78 & 3.21 \\
75-Minute Trial & 0.00 & 0.00 & \\
$\quad$ Before & & & \\
$\quad$ After & 15.27 & 80.78 & 1.45 \\
90-Minute Trial & 4.27 & 11.64 & \\
$\quad$ Before & After & & \\
Critical Values: $1.345(90 \%), 1.761(95 \%), 2.624(99 \%)$ & \\
\hline
\end{tabular}

\section{Conclusions}

By using a hay steamer, owners of livestock and equine can offer a healthier and safer alternative feed compared to hay exposed to quality decreasing variables. Hay quality changes due to methods of storage, time harvested, and other environmental variables. This experiment has shown that the portable hay steamer does have an impact on bacteria colony count which is beneficial to owners.

It is not economically feasible to discard hay due to mold or other undesirable qualities. Using a hay steamer offers an opportunity for owners to use stored hay that has been exposed to more variable conditions and gives a possible solution to help manage negative effects on the health of their animals due to mold. The use of a hay steamer is most beneficial when used on hay that is heavily populated with bacteria. The marginal benefit to using the steamer on fresh, clean bales is lower; however, the hay steamer can act as an insurance policy for the owner since one cannot tell the damage done by external factors on a microscopic level.

One issue that was not addressed in the current research is the scalability of steaming. Specifically, what is the quantity of hay to be steamed in each batch to ensure maximum efficiency of the steamer? Future research is needed to determine the points of maximum temperature in the steamer and calculate the amount of hay required to reach this capacity.

With the use of a hay steamer, animal owners can reduce costs associated with discarding hay and possibly increase performance and the health of their animals. Owners will not only be able to utilize their left over hay; but they will be able to utilize it in a simple, cost effective way. The hay steamer is most efficient at a short span of steaming time, 45-75 minutes. Longer steaming time does not improve efficiency past 75 minutes. There is very little labor involved in using the hay steamer and it is simple to use which makes it desirable to those who own smaller operations. By using a hay steamer, the owner can provide good quality hay, 
which will benefit the health of their animals as well as eliminate costs associated with wasted hay.

\section{Acknowledgement}

The authors would like to thank HayGain for the use of equipment and technical expertise.

\section{References}

Blackman, M., \& Moore-Colyer, M. J. (1998). Hay for horses: the effects of three different wetting treatments on. Animal Science, 745-750.

Clarke, A. (1992). Environmental monitoring in relation to Current Therapy in Equine Medicine, 310-315.

Doane, D. P., \& Seward L. E. (2007). Applied Statistics in Business and Economics, McGraw-Hill Irwin, 406-407.

Eduard, W., Lacey, J., Karlsson, K., Palmgren, U., \& Blomquist, G. (1990). Evaluation of methods for enumerating microorganisms in filter samples from highly contaminated occupational environments. American Industrial Hygiene Association, 427-436.

Gregory, P. H., \& Lacey, M. E. (1963). Mycological examination of dust from moldy hay associated with farmer's lung disease. Journal of General Microbiology, 75-88.

Hofve, J. (2007). Respiratory Conditions in Horses. [Online] Equina Wellness, Available: http://www.equinawellness.com/equine-respiratory.htm (July 30, 2015)

Moore-Colyer, M., \& James, R. (2009). The effect of steam treatment on the total viable count, mold and yeast numbers in hay using the Haygain Hay Steamer. Cirencester, Gloucestershire: Royal Agricultural College GL 7 6JS. [Online] Available: https://www.smartpakequine.com/pdfs/products/haygaintheeffectofsteamtreatment.pdf (July $30,2015)$

PM, D., DI, R., \& BC, M. (1995). Equine pulmonary disease: a case control study of 300 referred cases. Part 1: Examination techniques, diagnostic criteria and diagnoses. Equine Vet Journal, 416-421.

Warr, E., \& Petch, J. (1992). Effects of soaking hay on its nutritional quality. Equine Veterinary Education, 169-171.

\section{Copyright Disclaimer}

Copyright for this article is retained by the author(s), with first publication rights granted to the journal.

This is an open-access article distributed under the terms and conditions of the Creative Commons Attribution license (http://creativecommons.org/licenses/by/3.0/). 